\title{
All-optical manipulation of electron spins in carbon nanotube quantum dots
}

\author{
Christophe Galland and Ataç Imamoğlu \\ Institute of Quantum Electronics, ETH Zurich, Wolfgang-Pauli-Strasse 16, CH-8093 Zurich, Switzerland
}

(Dated: July 9, 2021)

\begin{abstract}
We demonstrate theoretically that it is possible to manipulate electron or hole spins all optically in semiconducting carbon nanotubes. The scheme we propose is based on the spin-orbit interaction that was recently measured experimentally; we show that this interaction, together with an external magnetic field, can be used to achieve optical electron-spin state preparation with fidelity exceeding $99 \%$. Our results also imply that it is possible to implement coherent spin rotation and measurement using laser fields linearly polarized along the nanotube axis, as well as to convert spin qubits into time-bin photonic qubits. We expect that our findings will open up new avenues for exploring spin physics in one-dimensional systems.
\end{abstract}

Optical manipulation of spins in atoms or semiconductors relies on the presence of strong spin-orbit interaction (SOI) either in the initial or final state of an optical transition. In III-V semiconductors, it is the large spin-orbit splitting of the valence band states [1] that enables efficient optical pumping of electron [2] or nuclear spins [3, 4] and leads to a strong correlation between light helicity and electron spin orientation [5]. In this context, one would argue that optical spin manipulation would be hindered in semiconducting single-wall carbon nanotubes (CNTs): due to the weak spin-orbit splitting in graphene [6] and early experiments suggesting the presence of electron-hole symmetry [7], it had been assumed that SOI in CNTs would be small for both electrons and holes. In addition, the depolarization effect ensures that only electric fields linearly polarized along the CNT axis couple strongly to electrons and holes [8, 9], ruling out the possibility of obtaining correlations between electron spin and photon polarization.

In this Letter, we describe a scheme for realizing efficient optical manipulation of spins in CNTs. Our work is motivated by the recent experimental observation of SOI-induced zerofield spin splitting in CNTs [10]. The breakdown of electronhole symmetry that is a consequence of finite SOI implies that a finite external axial magnetic field could be used to cancel the SOI-induced spin splitting of the hole, while retaining a finite splitting for the electron spin. The presence of a magnetic field component perpendicular to the CNT axis then mixes the hole spin states and allow for a very efficient spin-flip Raman coupling between the electron spin states. In addition to analyzing the spin pumping efficiency as a function of the external magnetic and laser fields, we discuss applications of the proposed scheme in quantum information processing.

The band structure of a CNT can be derived from that of graphene, in which conduction and valence bands are crossing at two inequivalent points in the reciprocal lattice (labeled $\mathrm{K}$ and $\mathrm{K}^{\prime}$ ) with linear dispersions. Since the K and $\mathrm{K}^{\prime}$ points are at the boundaries of the first Brillouin zone , the states near the energy gap in semiconducting CNTs have a large azimuthal momentum $k_{\perp}$. In a semi-classical picture, these states correspond to electrons having a fast clockwise or counter-clockwise circular motion around the CNT's circumference, therefore exhibiting an orbital magnetic moment $\mu_{\text {orb }} \approx 0.3 \cdot d[\mathrm{~nm}] \mathrm{meV} / \mathrm{T}$ pointing along the axis, with opposite signs for states originating from the two different valleys
[10, 11]. The degeneracy between these states is therefore lifted when a magnetic field $B_{\|}$is applied along the nanotube axis [11, 12]. The spin of electrons (or holes) also couples to the magnetic field, yielding a Zeeman splitting $\Delta_{Z}=g \mu_{B} B_{\|}$, with $g \approx 2$ for both electrons and holes [10]. In this simple picture the electronic states should be four-fold degenerate at zero magnetic field. Here, we focus on a CNT quantum dot (CNT QD) trapping a single electron, since this is the system of primary interest from a quantum information perspective [13].

The SOI leads to a zero-field spin splitting $\Delta_{S O}$ between states with parallel and anti-parallel spin and orbital magnetic moments [10, 18] (Fig. 1a). In the following analysis, we rely on the experimentally measured values for $\Delta_{S O}$ [10]. Since we are interested in optical manipulation of spins, we consider a QD formed on a semiconducting CNT with a diameter $d \sim 1.2 \mathrm{~nm}$ having its lowest optical transition in the nearinfrared $(\sim 1500 \mathrm{~nm})$ [19]. Extrapolating measured values from [10, 11] to this diameter $\left(\mu_{\text {orb }} \propto d\right.$ and $\left.\Delta_{S O} \propto \frac{1}{d}\right)$ we expect $\mu_{\text {orb }} \approx 0.36 \mathrm{meV} / \mathrm{T}, \Delta_{S O}^{e} \approx 1.5 \mathrm{meV}$ for electrons and $\Delta_{S O}^{h} \approx 0.9 \mathrm{meV}$ for holes [20].

We first study the possibility of optical spin pumping using resonant laser fields. In Fig. 1a we show the energy level diagram of the lowest electron and hole states in a CNT QD under an axial magnetic field $B_{\|}$as confirmed experimentally by Kuemmeth et al. [10]. We will label U (D) the states having a positive (negative) orbital magnetic moment. These states originate from the two different valleys $\mathrm{K}$ and $\mathrm{K}^{\prime}$ and mix very weakly in clean CNTs $\left(\Delta_{K K^{\prime}} \approx 65 \mu \mathrm{eV}\right.$ in [10]). Allowed optical transitions are of the type $\mathrm{U} \rightarrow \mathrm{U}$ or $\mathrm{D} \rightarrow \mathrm{D}$ due to momentum conservation. The up and down arrows represent the projection of the spin along the CNT axis ( $\uparrow$ for $S_{z}=+\frac{\hbar}{2}$ ) and the subscripts designate electron or hole states. We now apply an axial magnetic field $B_{\|}$and a laser field polarized linearly along the nanotube axis, strongly coupling states from the same valley with opposite electron and hole spins. In addition, we assume the presence of a magnetic field component $B_{\perp}$ orthogonal to the CNT axis that coherently mixes the up and down electron (and hole) spin states.

Figure 1b shows an energy level diagram equivalent to that of Fig. 1a in the trion picture [2] where the four lowest energy spin states of a CNT QD as well as the optically excited states with two electrons and one hole are depicted 

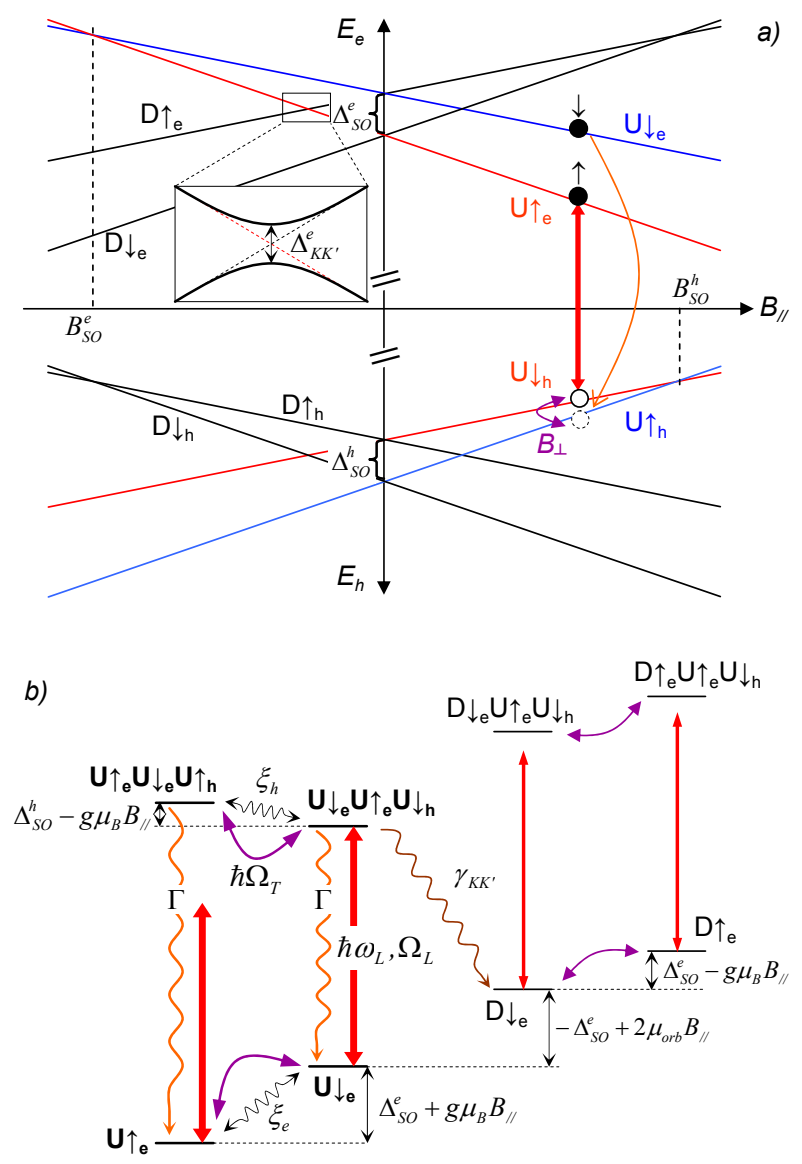

FIG. 1: a) Energy diagram of the lowest electron (subscript $e$ ) and hole (subscript $h$ ) states in a nanotube quantum dot as a function of the applied axial magnetic field $B_{/ /[10,18]}$. b) Energy diagram of a singly charged nanotube quantum dot for large $B / /$ showing the relevant optical transitions coupled by a laser field polarized along the CNT axis with Rabi frequency $\Omega_{L}$. The orthogonal magnetic field causes a coherent $\uparrow-\downarrow$ coupling with strength $\hbar \Omega_{T}=g \mu_{B} B_{\perp}$. The decay of the excited states is assumed to be spin-conserving and mono-exponential with rate $\Gamma$. Spin relaxation rates for electrons and holes are denoted by $\xi_{e}$ and $\xi_{h}$. K-K' mixing can cause optically assisted valley-flip to the state $\mathrm{D} \downarrow_{e}$ at an effective rate $\gamma_{K K^{\prime}}$.

[21]. We choose the energy of the laser to be resonant with the $\mathrm{U} \downarrow_{e} \rightarrow \mathrm{U} \downarrow_{e} \mathrm{U} \uparrow_{e} \mathrm{U} \downarrow_{h}$ transition. The optically excited trion state now couples to $\mathrm{U} \downarrow_{e} \mathrm{U} \uparrow_{e} \mathrm{U} \uparrow_{h}$ because the hole spin precesses around the perpendicular field $B_{\perp}$. Radiative recombination from $\mathrm{U} \downarrow_{e} \mathrm{U} \uparrow_{e} \mathrm{U} \uparrow_{h}$ leaves a spin-up electron $\mathrm{U} \uparrow_{e}$. Since the optical transition $\mathrm{U} \uparrow_{e} \rightarrow \mathrm{U} \downarrow_{e} \mathrm{U} \uparrow_{e} \mathrm{U} \uparrow_{h}$ is detuned by $\Delta_{S O}^{e}+\Delta_{S O}^{h}$ from the applied laser field, light scattering (experimentally measured by laser absorption) will vanish [2], ensuring that the resident electron spin remains in state $\mathrm{U} \uparrow e$. Conversely, preparation of the spin in the $\mathrm{U}_{\downarrow}$ state can be achieved by tuning the laser field onto resonance with the $\mathrm{U} \uparrow_{e} \rightarrow \mathrm{U} \downarrow_{e} \mathrm{U} \uparrow_{e} \mathrm{U} \uparrow_{h}$ transition.

To assess the efficiency of optical spin pumping as a function of the applied magnetic and laser fields we have performed numerical simulations using the optical Bloch equa- tions for the 4-level system shown in the left part of Fig. $1 \mathrm{~b}$. We ignore K-K' mixing for the time being. The spin-flip rates $\xi_{e}\left(\xi_{h}\right)$ for electrons (holes) are dominated by phononassisted spin relaxation [18, 23]: these rates are expected to have magnitudes varying from $1 \mu \mathrm{s}^{-1}$ to $1 \mathrm{~ms}^{-1}$. We take the exciton recombination time to be $\Gamma^{-1} \approx 40 \mathrm{ps}$, corresponding to the photoluminescence (PL) lifetime measured on individual CNTs [14, 15]. The narrowest reported nanotube PL linewidths $(0.25-0.5 \mathrm{meV}[12,24])$ however, are an order of magnitude larger than the lifetime broadening. We therefore include a Markovian dephasing rate of both optical transitions $\hbar \gamma_{\text {deph }}=0.25 \mathrm{meV}$.

The resulting spin population imbalance as a function of the axial and perpendicular components of the magnetic field is shown in Fig. 2a, when the laser is resonant with the red (lowest energy) transition. First, we remark that spin preparation with a fidelity close to 1 is possible at almost any axial field provided that the perpendicular field is of the order of a few $100 \mathrm{mT}$. But the main feature revealed by the simulation is the peculiar behavior of the system when the Zeeman splitting caused by $B_{\|}$cancels the spin-orbit splitting for either electrons or holes: when $B_{\|}=B_{S O}^{h}=\Delta_{S O}^{h} /\left(g \mu_{B}\right)$, the states $\mathrm{U} \downarrow_{e} \mathrm{U} \uparrow_{e} \mathrm{U} \downarrow_{h}$ and $\mathrm{U} \downarrow_{e} \mathrm{U} \uparrow_{e} \mathrm{U} \uparrow_{h}$ have the same energy; as a consequence the mixing induced by even a vanishingly small $B_{\perp}$ suffices to yield very efficient electron spin pumping. On the contrary, for $B_{\|}=B_{S O}^{e}=\Delta_{S O}^{e} /\left(g \mu_{B}\right)$, the resonance occurs between the electronic states and the electron spin remains randomized for all values of $B_{\perp}$.

Since it is likely that the excited trion states undergo faster dephasing than the single electron ground states, we performed an additional simulation where the coherence between the two trion states is also dephased at the rate $\hbar \gamma_{d e p h}$. The result, shown in Fig. 2b, is qualitatively similar to the case depicted in Fig. 2a. For $B_{\|} \approx B_{S O}^{h}$ and $B_{\perp}=0.2 \mathrm{~T}$ (inset Fig. 2b) very efficient spin preparation in either state $\mathrm{U} \uparrow_{e}$ or $\mathrm{U} \downarrow_{e}$ is achieved upon tuning the laser across the $\mathrm{U} \downarrow_{e} \rightarrow \mathrm{U} \downarrow_{e} \mathrm{U} \uparrow_{e} \mathrm{U} \downarrow_{h}$ and $\mathrm{U} \uparrow_{e} \rightarrow \mathrm{U} \downarrow_{e} \mathrm{U} \uparrow_{e} \mathrm{U} \uparrow_{h}$ transitions. Pauli blockade leads to a drop in absorption by more than an order of magnitude: this would be the experimental signature of spin pumping in differential transmission measurements [2]. Our results demonstrate that selective optical spin preparation in CNT QDs is in experimental reach. A limitation would however appear for very small QDs $(\sim 10 \mathrm{~nm})$ : it was shown recently that the ohmic coupling of strongly confined excitons to one-dimensional acoustic phonons in CNTs leads to asymmetric absorption spectrum with a pronounced blue tail, extending over a few $\mathrm{meV}$ [15]. This pure-dephasing process would nevertheless not alter the efficiency of spin pumping when driving the lower-energy resonance with a red-detuned laser.

The presence of K-K' valley mixing (characterized by the splitting $g \mu_{B} B_{K K^{\prime}}=\Delta_{K K^{\prime}}$ ) will result in a finite probability that the electron spin leaves the Hilbert space spanned by $\mathrm{U} \downarrow_{e}$ and $\mathrm{U} \uparrow_{e}$. We denote the effective spin-flip Raman scattering rate from state $\mathrm{U} \downarrow_{e}$ to $\mathrm{U} \uparrow_{e}$ with $\gamma_{\uparrow \downarrow}$ and the effective rate for a laser assisted transition from $\mathrm{U} \downarrow_{e}$ to $\mathrm{D} \downarrow_{e}$ with $\gamma_{K K^{\prime}}$. Us- 


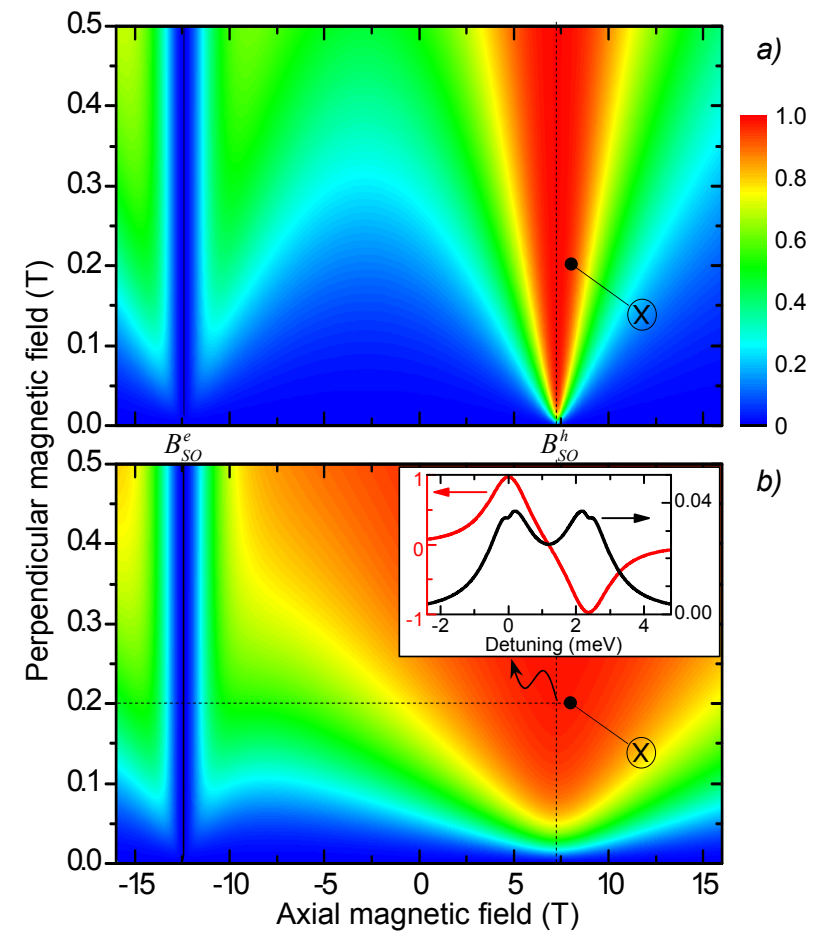

FIG. 2: The contour plot of the population difference between states $\mathrm{U} \uparrow_{e}$ and $\mathrm{U} \downarrow_{e}$ as a function of the magnetic field components when the laser is kept resonant with the $\mathrm{U} \downarrow_{e} \rightarrow \mathrm{U} \downarrow_{e} \mathrm{U} \uparrow_{e} \mathrm{U} \downarrow_{h}$ transition (zero of the detuning scale in the inset). We take $\xi_{e}=\xi_{h}=0.1 \mu \mathrm{s}^{-1}$ and $\Gamma^{-1}=40$ ps. In (a), we assume that the optical transitions are broadened by Markovian dephasing with rate $\hbar \gamma_{d e p h}=0.25 \mathrm{meV}$. In (b), we consider a scenario where the coherence between states $\mathrm{U} \downarrow_{e} \mathrm{U} \uparrow_{e} \mathrm{U} \downarrow_{h}$ and $\mathrm{U} \downarrow_{e} \mathrm{U} \uparrow_{e} \mathrm{U} \uparrow_{h}$ also undergoes fast dephasing at the same rate $(0.25 \mathrm{meV})$. In the inset to part (b), we show the laser absorption (black line) and population difference (red line) between $\mathrm{U} \uparrow_{e}$ and $\mathrm{U} \downarrow_{e}$ states for external magnetic fields $B_{\|}=B_{S O}^{h}$ and $B_{\perp}=0.2 \mathrm{~T}$. The absorption (right scale) is normalized to its maximum value in absence of spin pumping (when $B_{\perp}=0 \mathrm{~T}$ ).

ing rate equations we obtain: $\frac{\gamma_{\uparrow \downarrow}}{\gamma_{K K^{\prime}}} \approx \frac{B_{\perp}^{2} /\left(\Delta_{S O}^{h}-g \mu_{B} B_{\|}\right)^{2}}{B_{K K^{\prime}}^{2} /\left(\Delta_{S O}^{e}-2 \mu_{o r b} B_{\|}\right)^{2}}$ which is maximum when $B_{\|} \rightarrow B_{S O}^{h}$. We find that using the experimentally measured parameters, efficient spin pumping is possible in large regions of magnetic fields. For example at point $\mathrm{X}$ in Fig. 2, for which $B_{\|}=8 \mathrm{~T} \approx B_{S O}^{h}+0.75 \mathrm{~T}$ and $B_{\perp}=0.2 \mathrm{~T}$, we have $\frac{\gamma_{\uparrow \downarrow}}{\gamma_{K K^{\prime}}}>100$. Once the system goes through a valley-flip Raman scattering to state $\mathrm{D} \downarrow_{e}$, the applied laser field will be detuned from the transition to the state $\mathrm{D} \downarrow_{e} \mathrm{U} \uparrow_{e} \mathrm{U} \downarrow_{h}$, due to the exchange terms of Coulomb interaction (see Fig. 1b, right). If $B_{K K^{\prime}}$ is large it would therefore be necessary to use a second re-pumping laser on this transition to reintroduce the electron to the U-valley.

In most experiments it has been observed that the lifetime of excitons is more than an order of magnitude shorter than the predicted radiative lifetime [14, 15, 25]. While radiative broadening can be enforced by embedding CNTs in cavity structures with a large Purcell factor [26], understanding the nature of non-radiative relaxation is crucial for identifying the limits of optical spin manipulation. In particular, if this relaxation is not spin-conserving, then spin pumping becomes efficient for an even larger range of applied magnetic field strengths. Most probable mechanisms for fast non-radiative decay proposed so far are phonon-assisted relaxation and/or multi-particle Auger processes [27]. Since these processes are spin-conserving, they will not alter the efficiency of spin pumping.

Having demonstrated that it is viable to prepare a single spin optically, we turn to coherent spin rotation and spin measurement. By using two laser fields satisfying two-photon Raman resonance condition under the same external magnetic field configurations that allow for efficient spin pumping, we can implement deterministic spin rotation [5]. To realize alloptical spin measurement, the field $B_{\perp}$ mixing the electron (hole) spin states must be turned off. In this limit, presence or absence of light scattering (or absorption) upon excitation by a resonant laser conveys information about the spin state [5]. For spin measurements, minimizing spin-flip non-radiative relaxation and inter-valley scattering is crucial. We also point out that all of our results would apply for a single-hole charged CNT QD as well.

Next, we address the possibility of transferring quantum information stored in the CNT QD electron spin to a generated photon. Given that the polarization of the photon is fixed by the geometry, the logical choice is to use time-bin entanglement [28]. We assume that our CNT QD is coupled to an optical cavity whose energy $\omega_{\text {cav }}$ is resonant with the transition $\mathrm{U} \downarrow_{e} \rightarrow \mathrm{U} \downarrow_{e} \mathrm{U} \uparrow_{e} \mathrm{U} \downarrow_{h}$ (Fig. 3). Using combinations of laser pulses one can prepare an initial state in the coherent spin superposition: $\left|\psi_{i n}\right\rangle=\left(\alpha\left|\mathrm{U} \uparrow_{e}\right\rangle+\beta\left|\mathrm{U} \downarrow_{e}\right\rangle\right) \otimes\left|0_{c}\right\rangle$ where $\left|0_{c}\right\rangle$ is the empty cavity mode. We now send two well separated $\pi$-pulses at time $t_{1}$ and $t_{2}$ with respective energies $\omega_{a}$ and $\omega_{b}$ as shown in Fig. 3. We define the two creation operators $a^{\dagger}$ $\left(b^{\dagger}\right)$ for cavity-mode photons emitted immediately after pulse 1 (pulse 2). The optical transition at frequency $\omega_{b}$ is allowed because of the mixing induced by $B_{\perp}$, and the rates of both transitions can be made identical by adjusting the pulse intensities. The first pulse excites the trion state if and only if the spin is initially down. In this case Purcell effect ensures very fast spontaneous emission and projection onto the state $\left|\mathrm{U} \downarrow_{e}\right\rangle \otimes a^{\dagger}\left|0_{c}\right\rangle$. If the spin is initially up, the transition is Pauliblocked and we are left with $\left|\mathrm{U} \uparrow_{e}\right\rangle \otimes\left|0_{c}\right\rangle$. The initial state has thus evolved to: $\left|\psi_{1}\right\rangle=\alpha\left|\mathrm{U} \uparrow_{e}\right\rangle \otimes\left|0_{c}\right\rangle+\beta\left|\mathrm{U} \downarrow_{e}\right\rangle \otimes a^{\dagger}\left|0_{c}\right\rangle$. We can do the same analysis for the second pulse and find that the final state is: $\left|\psi_{f}\right\rangle=\left|\mathrm{U} \downarrow_{e}\right\rangle \otimes\left(\alpha b^{\dagger}+\beta a^{\dagger}\right) \otimes\left|0_{c}\right\rangle$ where quantum information has been mapped onto a photon time-bin qubit. We emphasize that time-bin qubits are promising candidates for long range quantum communication using optical fibers [29] and that CNTs can be chosen to emit in the desired wavelength window.

We remark that one of the most interesting perspectives enabled by the considerations of this letter is the study of nuclear spin physics. The possibility of electron spin pumping should allow for the optical manipulation of nuclear spin ensembles, which has been successfully achieved in GaAs-based struc- 

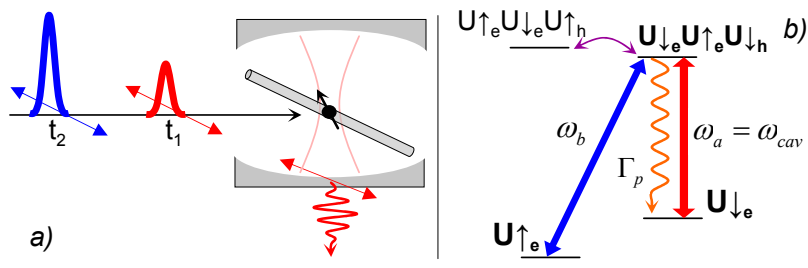

FIG. 3: a) Schematics of the cavity QED setup discussed in the text. Both laser pulses are polarized along the nanotube axis. Provided that the pulse separation $t_{2}-t_{1}$ is larger than all the other time scales (inverse Rabi frequency and cavity-enhanced exciton decay rate) quantum information can be efficiently encoded in a photon time-bin qubit. b) Energy diagram of the nanotube quantum dot with the relevant transitions and rates used in the scheme.

tures [3, 4]. However, experimental knowledge of the strength and characteristics of hyperfine interaction in CNTs is still lacking. Of particular interest in this context would be dynamic nuclear spin polarization in a CNT QD where hundreds or thousands of ${ }^{13} \mathrm{C}$ atoms would form an ideal $I=\frac{1}{2}$ spin bath. Alternatively, using high-purity ${ }^{12} \mathrm{C} C N T s$, one may realize QDs interacting with only 1 or 2 nuclear spins [30].

This work was supported by a grant from the Swiss National Science Foundation (SNSF).

[1] P. Y. Yu and M. Cardona, Fundamentals of Semiconductors, p 71-80 and 480-486 (Springer-Verlag, Berlin, 2003).

[2] M. Atatüre et al., Science 312, 551 (2006).

[3] C. W. Lai et al., Phys. Rev. Lett. 96, 167403 (2006).

[4] D. Gammon et al., Science 277, 85 (1997).

[5] A. Imamoglu et al., Phys. Rev. Lett. 83, 4204 (1999).

[6] Y. Yao et al., Phys. Rev. B 75, 041401 (2007).

[7] P. Jarillo-Herrero et al., Nature 429, 389-392 (2004).

[8] S. Uryu and T. Ando, Phys. Rev. B 74, 155411 (2006).

[9] J. Lefebvre et al., Phys. Rev. Letter 98, 167406 (2007).

[10] F. Kuemmeth et al., Nature 452, 448-452 (2008).

[11] E. D. Minot et al., Nature 428, 536-539 (2004).

[12] A. Srivastava et al., Phys. Rev. Letter 101, 087402 (2008).
[13] Our scheme requires that the charged exciton (trion) be trapped as well. We note that the recent experiments demonstrated strong unintentional confinement of nanotube excitons [14] 15], possibly around charged defects. Enhanced interactions in 1D systems should lead to a trion binding energy well exceeding several meVs, suggesting that external gates could confine trions as well as electrons, even when a neutral exciton is not confined. Alternatively, local strain was shown to shift the excitonic states very efficiently [16, 17] and could be used for trion localization.

[14] A. Högele et al., Phys. Rev. Lett. 100, 217401 (2008).

[15] C. Galland et al., Phys. Rev. Lett. 101, 067402 (2008).

[16] H. Maki et al., Nano Lett. 7, 890-895 (2007).

[17] Yang et al., Phys. Rev. Lett. 85, p154 (2000).

[18] D. B. Bulaev, B. Trauzettel and D. Loss, Phys. Rev. B 77, 235301 (2008).

[19] R. B. Weisman and S. M. Bachilo, Nano Lett. 3, 1235-1238 (2003).

[20] The discrepancy between $\Delta_{S O}^{e}$ and $\Delta_{S O}^{h}$ found in [10] is theoretically unexplained and seemingly dependent on experimental conditions.It is neither significant nor necessary for the scheme that we propose.

[21] In a neutral CNT, excitons from the two valleys mix and form symmetric and antisymmetric combinations: among the 16 possible valley and spin combinations, only the singlet-spin symmetric state is optically active [22]. In the presence of a confined $\mathrm{U} \downarrow_{e}$ electron, Pauli exclusion prevents the formation of a valley symmetric optical excitation. Since the Coulomb exchange interaction that couples $\mathrm{U} \downarrow_{e} \mathrm{D} \uparrow_{e} \mathrm{D} \downarrow_{h}$ and $\mathrm{U} \downarrow_{e} \mathrm{D} \downarrow_{e} \mathrm{D} \uparrow_{h}$ is stronger than the intervalley mixing [22], we expect the $\mathrm{U} \downarrow_{e} \mathrm{U} \uparrow_{e} \mathrm{U} \downarrow_{h}$ to form a bright state detuned from the other optically excited spin-singlet trion state by several meV.

[22] M.S. Dresselhaus et al., Annu. Rev. Phys. Chem. 58, 719-747 (2007).

[23] K.M. Borysenko et al., Phys. Rev. B 77, 205402 (2008).

[24] H. H'toon et al., Phys. Rev. Lett. 93, 027401 (2004).

[25] H. Hirori et al., Phys. Rev. Lett. 97, 257401 (2006).

[26] A. Badolato et al., Science 308, 1158 (2005).

[27] V. Perebeinos and P. Avouris, Phys. Rev. Lett. 101, 057401 (2008).

[28] J. Brendel et al., Phys. Rev. Lett. 82, 2594 (1999).

[29] I. Marcikic et al., Phys. Rev. Lett. 93, 180502 (2004).

[30] L. Childress et al., Science 314, 281 (2006). 